\title{
Cocoon Filament Quality of a Special Silkworm Variety, GoldenSilk
}

\author{
HaeYong Kweon*, Kwang-Gill Lee, KwangYoung Park, Seok Woo Kang, Pil-Don Kang, Mi-Ja Kim, and \\ Kee Young Kim \\ Sericultural \& Apicultural Materials Division, National Academy of Agricultural Science, RDA, Suwon 441-100, Republic of \\ Korea
}

(Received 10 October 2011; Accepted 20 February 2012)

The effect of cultivation season on the cocoon filament quality of authorized silkworm variety, GoldenSilk was examined with cocoon filament character, reeling character, and fiber character during 3 years. In cocoon filament character, filament length of spring and autumn cocoon was 979 and $879 \mathrm{~m}$, respectively. Cocoon filament weight of spring and autumn cocoon was 33.1 and $26.5 \mathrm{cg}$, which is lower than that of general bivoltine strain, BaeGokJam. Raw silk percentage of spring and autumn cocoon was 15.65 and $13.77 \%$, respectively. In reeling character, reelability showed similar trend regardless of cultivation season and strain. However, the length and weight of non-broken filament of spring season were slightly higher than those of autumn cocoon. Cultivation season did not affect on the fiber characteristics such as clearness and neatness, lousiness, and degumming loss. In cocoon character, filament length, size, and weight, spring cocoon filament showed higher value than autumn cocoon, but lower than authorized general silkworm varieties. In reeling characteristics, non-broken length and weight showed the similar trends with the cocoon filament character. However, the reelability of GoldenSilk cocoon was not affected by cultivation season. The reelability of GoldenSilk cocoon was also similar with that of authorized general one. Fiber character including clearness and neatness, lousiness, and degumming loss was also similar regardless of the rearing season and different strain.

Key words: Cocoon filament quality, Cultivation season, Cocoon filament, Reeling

\footnotetext{
*To whom the correspondence addressed

Sericultural \& Apicultural Materials Division, National Academy of Agricultural Science, RDA, Suwon 441-100, Korea.

Tel: +82-31-290-8509; Fax: +82-31-290-8516;

E-mail: hykweon@korea.kr
}

\section{Introduction}

In general, cocoon has been used to make a textile fiber and surgical suture for several thousand years. Silk fiber is white and it is good for various dyeing. In textile industry, dyeing process is necessary to increase the value of the silk fabrics. During this process, dye waste water is released as unwanted pollution source. According to the needs, GoldenSilk strain with natural beautiful yellow color has been developed (Kang et al., 2007).

The productivity of silkworm is dependent on the climate variation. The climate variation affects on the quality of mulberry leave and rearing environment of silkworm breeds. In general, spring season is considered as the best season for silkworm rearing due to its favorable climate and good quality mulberry for rearing silkworm. In summer and autumn, there is high temperature and humidity, poor quality mulberry leaf that is not good for rearing silkworm. In Japan, silkworm rearing was conducted only during spring season till 1887 and thereafter extended even to summer and autumn seasons (Veldtman, 2002). Korean scientists have successfully developed season specific silkworm breeds for Korean climate. Some silkworm breeds such as ChunSuJam (Kang et al., 2001) and KumHwangJam (Kang et al., 2004) are recommended as spring season adaptable silkworm breeds. DaeSungJam (Sohn et al., 1987), ChuGangJam (Kang et al., 2003) and HaChoJam (Kang et al., 2002) have been nominated for summer-autumn season adaptable silkworm varieties. The traditional goal of the silkworm breeding is to breed a new silkworm variety for high silk yielding but that has been progressed to special interest and purpose for example, silkworm for Dongchunghacho (Kang et al., 2002). GoldenSilk variety recently developed by National Academy of Agricultural Science, RDA, Korea (Kang et al., 2007) would be expected to apply for value-added textile resources such as traditional Korean dress Hanbok, cos- 
tume for the dead, and so on. However, the seasonal effect on the quality of cocoon filament was not reported yet.

In the present study, we examined seasonal effect on cocoon filament quality such as silk filament length and weight per cocoon, size of cocoon filament, percentage of raw silk, non-broken filament weight and length, reelability of cocoon, degumming loss percentage, clearness and neatness, and lousiness from 2006 to 2009.

\section{Materials and Methods}

\section{Materials}

Silkworm (Bombyx mori L.) races, GoldenSilk (a special Korean authorized bivoltine variety) and BaeGokJam (a general Korean authorized bivoltine strain) were used for the present experiment. The larvae were fed exclusively on fresh mulberry leaves and cultivated at $25 \pm 1{ }^{\circ} \mathrm{C}$ with a humidity of $70-90 \%$. Sodium oleate and sodium carbonate was purchased and used without further purification.

\section{Characterization of cocoon quality}

For assessing the quality of the cocoons, the length, size, and weight of cocoon filament were determined according to the experimental guideline of sericulture (Kang et al., 2010). Cocoon was reeled using automatic reeling machine with $160 \mathrm{~m} / \mathrm{min}$.

\section{Clearness and neatness test and lousiness}

Cocoon filament fiber was wound on a panel and checked the note compared with standard panel. Fiber dyed with methylene blue was wound on the panel and compared with the standard picture.

\section{Degumming loss}

Cocoon was sliced and degummed as following method. One hundred fifty grams of cocoon was soaked into the degumming solution (sodium oleate $0.75 \mathrm{~g}$, sodium carbonate $0.45 \mathrm{~g}$ ) at boiling temperature for 40 minutes and then washed with distilled water. The degumming process was repeated twice. Degumming loss percentage was calculated according to the following equation.

Degumming loss percentage $(\%)=(\mathrm{W} i-\mathrm{W} f) / \mathrm{W} i * 100$

Where, $\mathrm{W} i$ is initial weight of dry cocoon; $\mathrm{W} f$, final weight of dry cocoon.

\section{Results and Discussion}

Cocoon filament characters

Cocoon filament characters are the length, size and weight

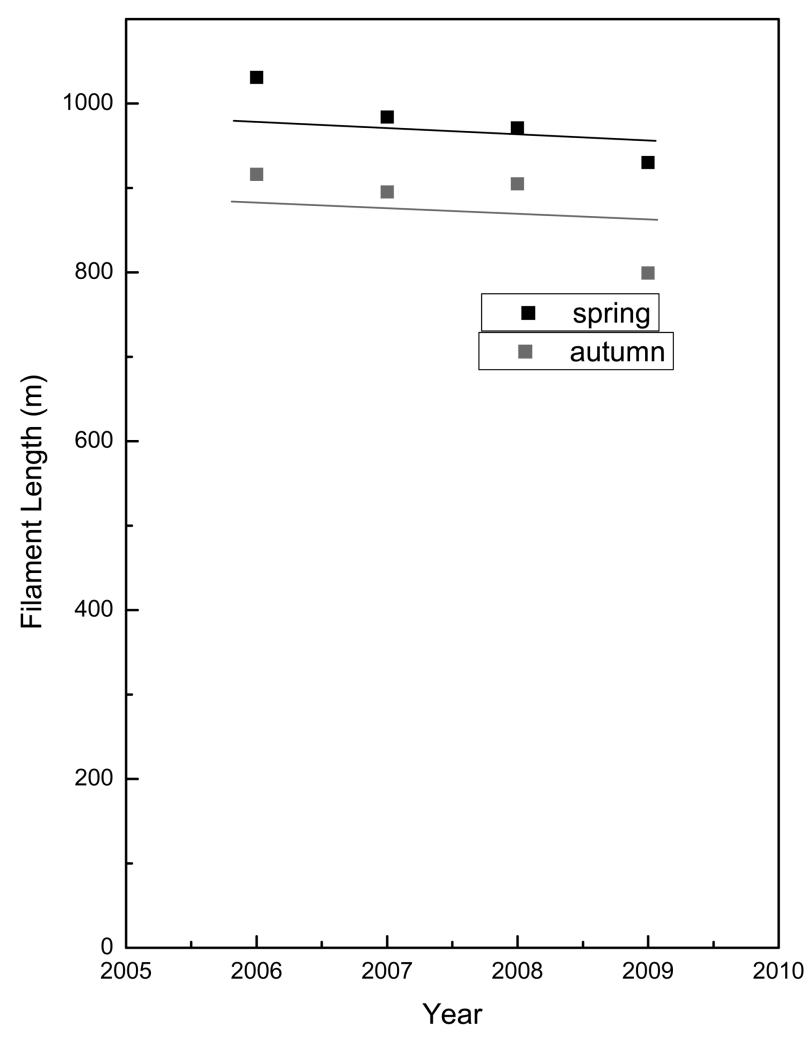

Fig. 1. Filament length of GoldenSilk cocoon.

of cocoon filament. The length of cocoon filament is the fibrous length reeled from a single cocoon. The average filament length of spring GoldenSilk cocoon was $979 \pm 42 \mathrm{~m}$ and that of autumn one was $879 \pm 54 \mathrm{~m}$ (Fig. 1). Length of spring cocoon is longer than that of autumn cocoon. Authorized silkworm variety, YangWonJam reared in spring 2009, spun $1622 \mathrm{~m}$ (Kang et al., 2011). The filament length of authorized general silkworm variety is longer than that of GoldenSilk strain for special use.

The average filament size of spring GoldenSilk cocoon was $3.06 \pm 0.21 \mathrm{~d}$ and that of autumn GoldenSilk cocoon was $2.72 \pm 0.09 \mathrm{~d}$ (Fig. 2). Size of spring cocoon is larger than that of autumn cocoon. Authorized silkworm variety, YangWonJam reared in spring 2009, was 2.72 denier (Kang et al., 2011). In general, the filament size range of authorized bivoltine variety in Korea is 2.50-3.00 denier. The filament size of GoldenSilk strain is adaptable for textile fiber.

The weight of cocoon filament is the weight of fibrous materials reeled from a single cocoon. The average filament weight of spring GoldenSilk cocoon was $33.1 \pm 1.51 \mathrm{cg}$ and that of autumn GoldenSilk cocoon was $26.5 \pm 1.02 \mathrm{cg}$ (Fig. 3). Filament weight of spring cocoon is heavier than that of autumn cocoon. Authorized silkworm variety, YangWonJam reared in spring 2009, was 


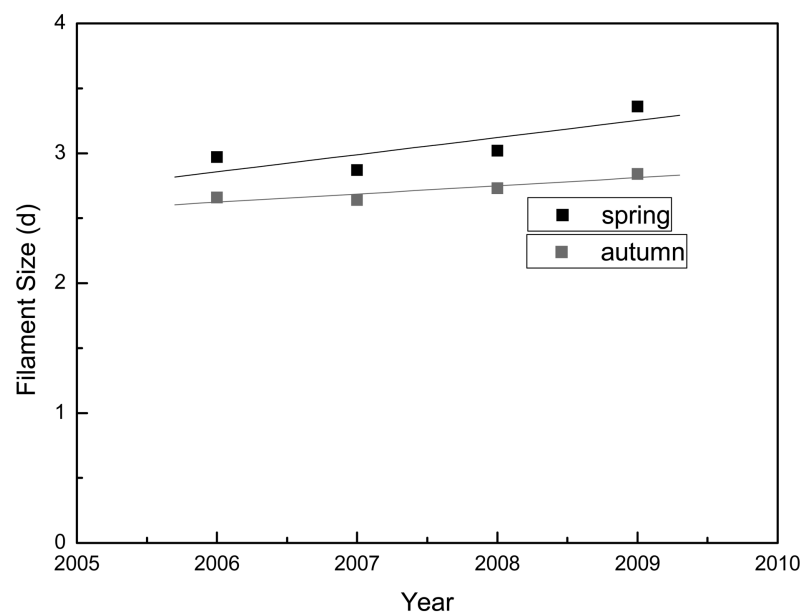

Fig. 2. Filament size of GoldenSilk cocoon.

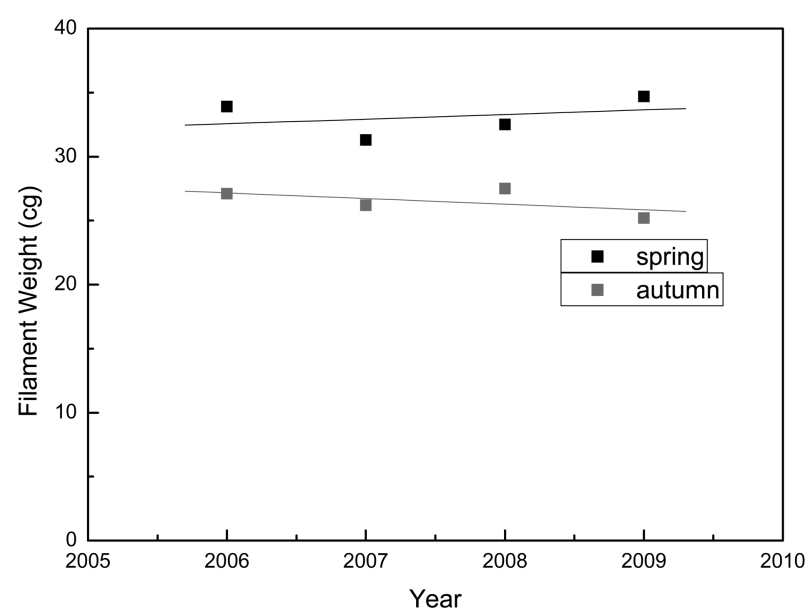

Fig. 3. Filament weight of GoldenSilk cocoon.

$48.9 \mathrm{cg}$ (Kang et al., 2011). The filament weight of authorized general silkworm variety is much higher than that of GoldenSilk variety.

Percentage of raw silk is one of major factor for cocoon character. Percentage of raw silk is defined as the percentage of raw silk weight against the whole cocoon weight. The raw silk percentage reared in spring season from 2006 to 2009 was $15.65^{\circ} æ 1.22$ and that in autumn season was $13.77^{\circ} æ 0.86$ (Fig. 4). In Korea, the government authorizes general healthy stains for farm rearing. Raw silk percentage of YangWonJam, one of current authorized silkworm strain, shows $20.81 \%$ (Kang et al., 2011). Park and Lee (1976) reported that cocoon beds have an influence on the percentage of raw silk and rotary cocoon bed was the best one. Percentage of raw silk of Golden silk cocoon is lower than that of authorized general strain. Spring cocoon showed higher raw silk percentage than autumn cocoon.

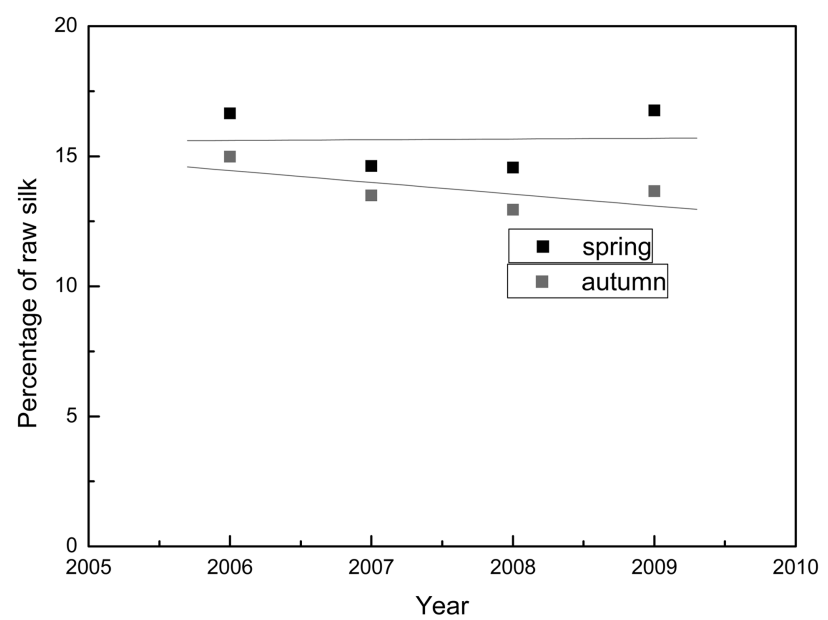

Fig. 4. Raw silk percentage of GoldenSilk cocoon.

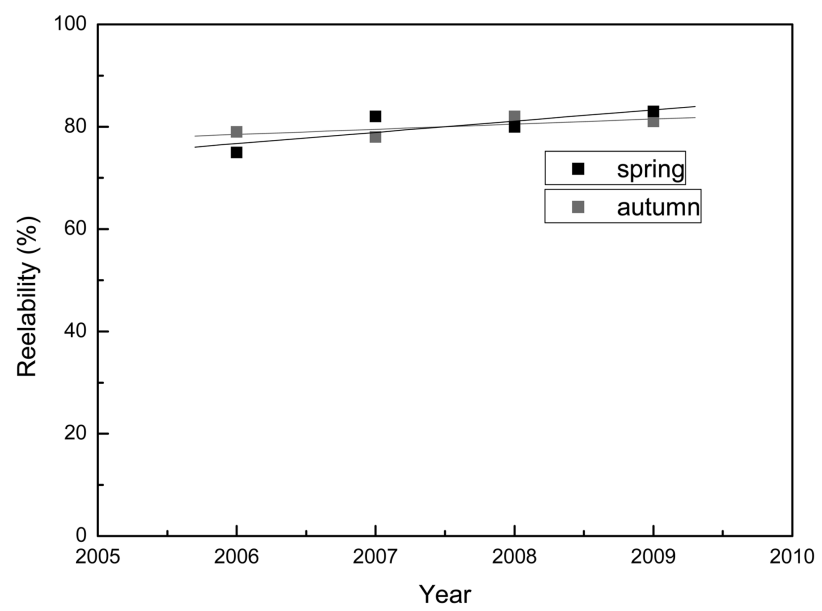

Fig. 5. Reelability of GoldenSilk cocoon.

\section{Reeling characters}

Fig. 5 showed the reelability of GoldenSilk variety during 3 years. The average reelability of GoldenSilk variety is $80 \%$. The general guideline for authorization of silkworm for textile fiber is over $70 \%$ reelability. GoldenSilk cocoon can be promising reelability for textile fiber.

Non-broken filament length is the total length of the continuous silk fiber reeled from a single cocoon without broken. Spring GoldenSilk cocoon has $782 \pm 19.2 \mathrm{~m}$ and autumn cocoon has $704 \pm 39.9 \mathrm{~m}$ (Fig. 6). The length of non-broken filament of spring cocoon is longer than that of autumn cocoon. Authorized bivoltine silkworm variety, YangWonJam reared in spring 2009, was $1309 \mathrm{~m}$ (Kang et al., 2011). Keshan and Ray (2000) reported that the length of unbroken silk filament of multiboltine strain was $314.8 \mathrm{~m}$.

Non-broken filament weight is the weight of the continuous silk fiber reeled from a single cocoon without bro- 


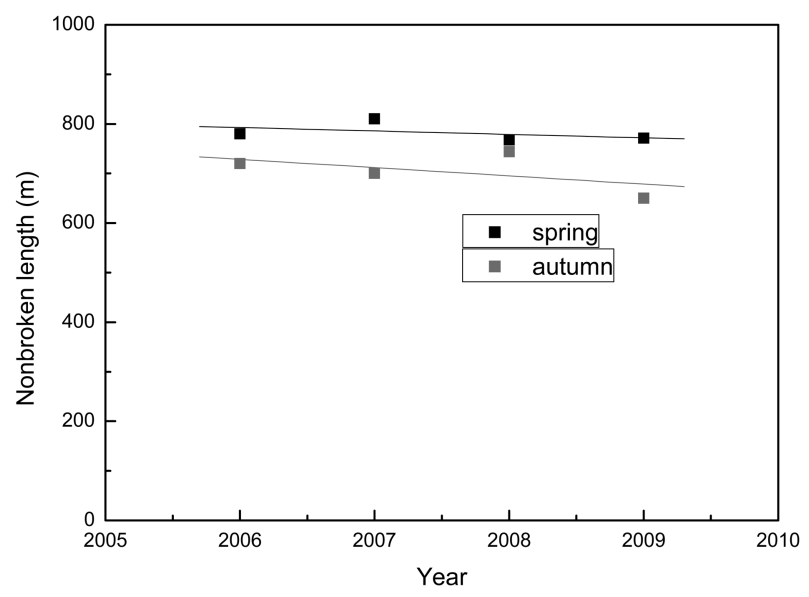

Fig. 6. Non-broken length of GoldenSilk cocoon.

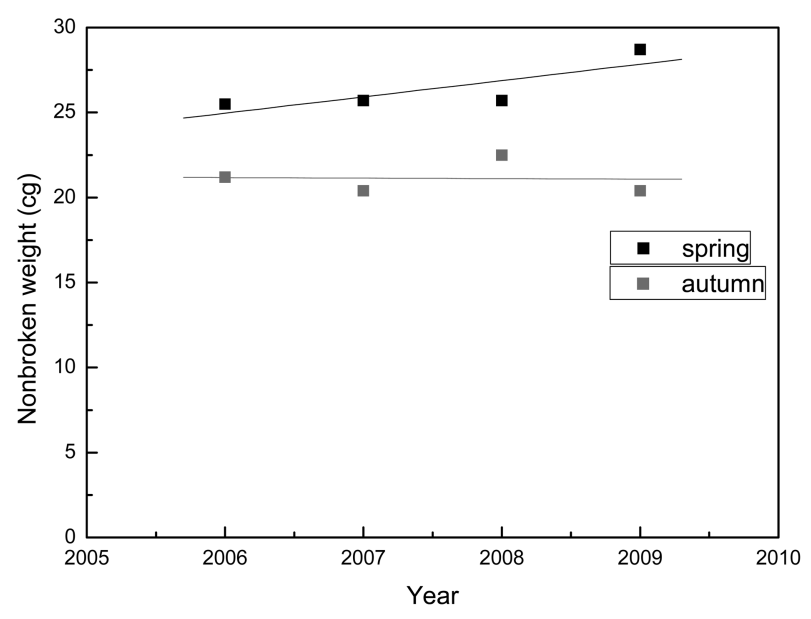

Fig. 7. Non-broken weight of GoldenSilk cocoon.

ken. Spring GoldenSilk cocoon has $26.4 \pm 1.54 \mathrm{cg}$ and autumn cocoon has $21.13 \pm 0.99 \mathrm{cg}$ (Fig. 7). Weight of non-broken filament of spring cocoon is heavier than that of autumn cocoon. Authorized bivoltine silkworm variety, YangWonJam reared in spring 2009, was $39.3 \mathrm{cg}$ (Kang et al., 2011).

\section{Fiber character}

Clearness and neatness of raw silk are important for textile fiber. The defects found in cocoon filament such as loops, split-ends, fuzziness, nibs, and hairiness affect directly raw silk quality. Spring cocoon and autumn cocoon showed similar values as $90 \pm 1.5$ and $90 \pm 2.8$ (Fig. 8 ). Clearness and neatness is known to be greatly influenced by mounting condition and handling of cocoon (Choi and Mah, 1970). Choi and Mah reported that the neatnesses of spring and autumn cocoon are similar each other. It is not recommended that silk varieties graded below 90 percent. The value of clearness and neatness is

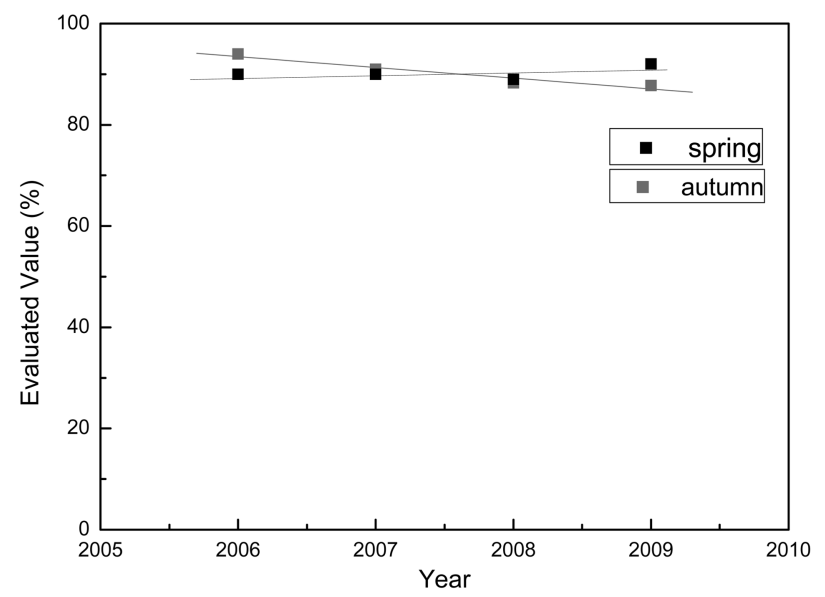

Fig. 8. Clearness and neatness of GoldenSilk cocoon.

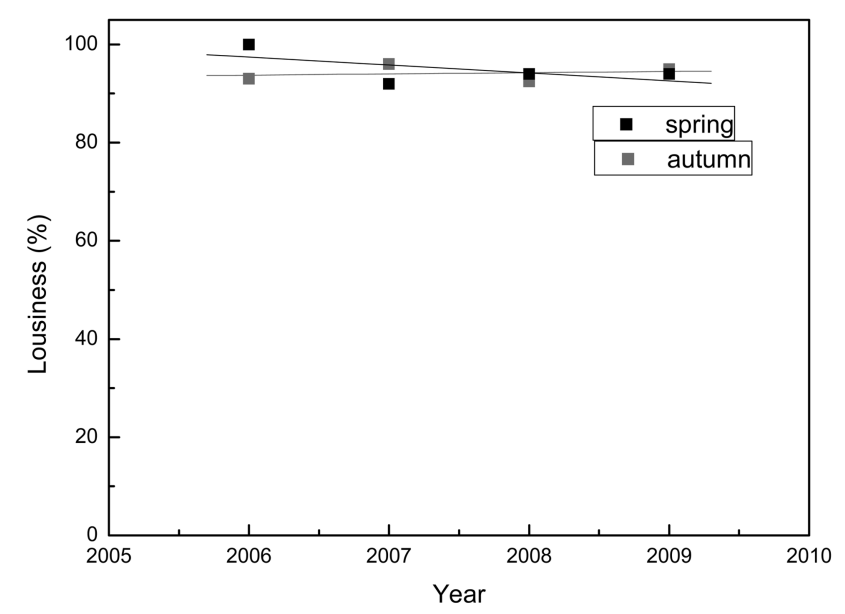

Fig. 9. Lousiness of GoldenSilk cocoon.

not influenced the rearing season for silkworm.

Lousiness in silk fabric is a severe problem in sericulture industry. Silk fabric with lousiness looks as if the fabric is covered with dust or is a paler shade than the rest. Lousiness is defects of silk fiber or fabric looks like hairlike projections, tiny entanglement of fibrils, and so on. Spring cocoon showed $95 \pm 3.5$ points and autumn cocoon $94 \pm 1.7$ points (Fig. 9). Lousiness is not significantly different between them.

Degumming process is essential for silk textile industry due to express silk characteristics including luster and softness. Cocoon filament is composed of fibroin and sericin. Through the degumming process, sericin is removed from the fibrous materials. The degumming loss percentage of GoldenSilk variety showed no significant different values among the rearing season; spring cocoon $25.4 \pm 0.3$, autumn cocoon $24.7 \pm 1.0$ (Fig. 10). Degumming loss of the authorized cocoon, YangWonJam and HanSaengJam, reared in spring 2009 showed $25.8 \%$ and $26.2 \%$, respec- 


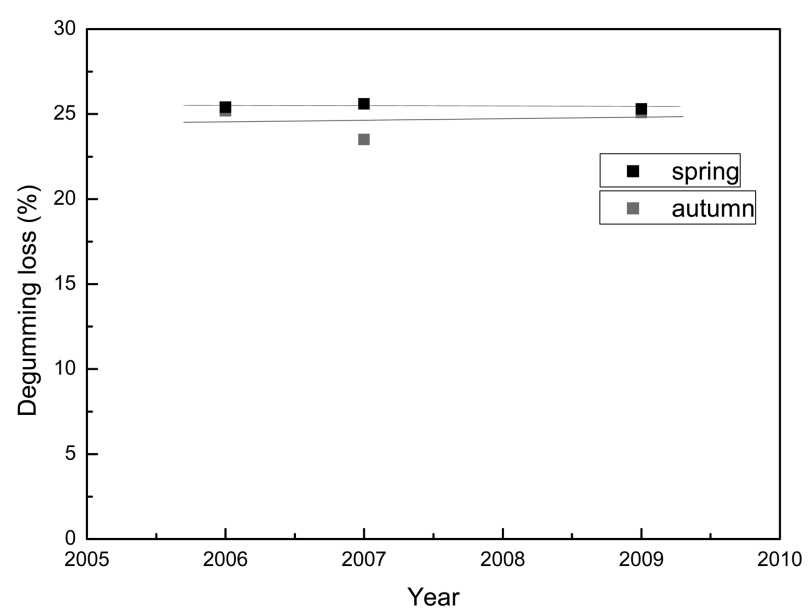

Fig. 10. Degumming loss of GoldenSilk cocoon.

tively (Kang et al., 2011). Degumming loss of GoldenSilk cocoon was similar to the authorized ones.

In cocoon filament character such as filament length, size, and weight, spring cocoon filament showed higher value than autumn cocoon, but lower than authorized general silkworm varieties. In reeling characteristics, nonbroken length and weight showed the similar trends with the cocoon filament character. However, the reelability of GoldenSilk variety is not affect by cultivation season. The reelability of GoldenSilk is also similar with that of authorized general bivoltine strain. Fiber character including clearness and neatness, lousiness, and degumming loss is also similar regardless of the rearing season and different strain.

\section{Acknowledgement}

This study was supported by the research grant (PJ007676042011) of Rural Development Administration (RDA) of Korea. The authors give special thanks to Kang, Pil-Don Kang, Mi-Ja Kim, Kee Young Kim for the advice on the silkworm varieties.

\section{References}

Choi BH, Mah SI (1970) The studies on various causes of clearness defects with in raw silk. Seri J Korea 12, 21-36.

Kang PD, Kim KM, Shon BH, Lee SU, Woo SO, Hong SJ (2001) Breeding of a new silkworm variety, ChunSuJam, with a high yielding for spring rearing season. Int J Indust Entomol 2, 65-68.

Kang PD, Shon BH, Lee SU, Hong SJ (2002) Breeding of a new non-cocooning silkworm variety, HaChoJam, suitable for autumn rearing season. Int J Indust Entomol 4, 77-81.

Kang PD, Shon BH, Lee SU, Woo SO, Hong SJ (2003) Breeding of a new silkworm variety, ChuGangJam, with a sex-limited larval making and high yielding for summer-autumn rearing season. Int J Indust Entomol 6, 57-61.

Kang PD, Shon BH, Lee SU, Kim MJ, Jung IY, Kim YS, Kim YD, Lee HS (2004) Breeding of a new silkworm variety, KumHwangJam, with a sex-limited cocoon color for spring rearing season. Int J Indust Entomol 9, 89-93.

Kang P, Lee S, Jung I, Shon B, Kim Y, Kim K, Kim M, Hong I, Lee K, Park K (2007) Breeding of new silkworm variety Golden Silk, a yellow cocoon color for spring rearing season. Korean J Seric Sci 49, 14-17.

Kang P, Sung G, Ji S, Kim M, Kweon H, Kang S (2010) Experimental guideline of sericulture (Korean), SangRokSa, Suwon.

Kang P, Sung G, Kim K, Lee H, Kim M, Ji S, Hong I, Kweon H, Park K, Lee K, Sohn B (2011) Breeding of HanSaengJam a parental sex-limited larval marking strain suitable for spring rearing season. Int J Indust Entomol 23, 179-182.

Keshan B, Ray AK (2000) Estradiol-17 $\beta$, in Bombyx mori: possible significance and its effect on silk production. $\mathrm{J}$ Insect Physiol 46, 1061-1068.

Park KJ, Lee SP (1976) Cocoon quality and economical advantages with the various cocoon beds. Seri J Korea 18, 95-100.

Sohn KW, Kim KM, Hong KW, Ryu KS, Choi SR, Mah YI, Kim KY, Lee SP, Kwon YH (1987) Breeding of "DaeSungJam", a sex-limited larval marking and high silk yielding silkworm variety for summer-autumn rearing season. Res Rept RDA 29, 54-60.

Veldtman R, McGeoch MA, Scholtz CH (2002) Variability in cocoon size in southern African wild silk moths: implications for sustainable harvesting. Afr Entomol 10, 127-136. 